University of Wollongong

Research Online

Faculty of Engineering and Information

Faculty of Engineering and Information

Sciences - Papers: Part A

Sciences

$1-1-2015$

Exact error performance analysis of binary space-time block coded cooperative communications systems in rayleigh fading channels

\author{
Le Chung Tran \\ University of Wollongong, Ictran@uow.edu.au \\ Zixuan Lin \\ University of Wollongong, z1715@uowmail.edu.au \\ Farzad Safaei \\ University of Wollongong, farzad@uow.edu.au \\ Alfred Mertins \\ University of Lubeck \\ Duy T. Ngo \\ University of Newcastle, duy.ngo@newcastle.edu.au
}

Follow this and additional works at: https://ro.uow.edu.au/eispapers

Part of the Engineering Commons, and the Science and Technology Studies Commons

Research Online is the open access institutional repository for the University of Wollongong. For further information contact the UOW Library: research-pubs@uow.edu.au 


\title{
Exact error performance analysis of binary space-time block coded cooperative communications systems in rayleigh fading channels
}

\begin{abstract}
Cooperative communications using the Alamouti space-time block code (STBC) has been mentioned intensively. However, to our knowledge, an in-depth error performance analysis for a space-time coded cooperative communications system, where each node acts as both a source node and a relay node for its partner, has not been reported. Intuitively, cooperative communications would not always be better than direct transmission, but only under certain conditions. This paper first derives an exact performance analysis of the Alamouti STBC cooperative communications for a Binary Phase Shift Keying (BPSK) constellation, which could be extended to a higher density modulation scheme. This mathematical analysis facilitates the source nodes to decide pro-actively whether cooperative communications or direct transmission should be used, depending on the channel conditions between the sources themselves and the channel conditions between the sources and the destination. The anterior knowledge of which transmission mode should be used before nodes actually engage in cooperation is useful to keep system operations as simple as possible, while assuring that cooperative communications is definitely beneficial once it is in place. The analytical error performances are then verified by simulations and in-depth discussions on the simulation results are presented.
\end{abstract}

\section{Keywords}

channels, fading, rayleigh, systems, communications, error, cooperative, exact, coded, block, time, space, binary, analysis, performance

\section{Disciplines}

Engineering | Science and Technology Studies

\section{Publication Details}

L. Tran, Z. Lin, F. Safaei, A. Mertins \& D. T. Ngo, "Exact error performance analysis of binary space-time block coded cooperative communications systems in rayleigh fading channels," in International Conference on Advanced Technologies for Communications (ATC), 2015, pp. 567-571. 


\section{Exact Error Performance Analysis of Binary Space-Time Block Coded Cooperative Communications Systems in Rayleigh Fading Channels}

\author{
Le Chung Tran, Zixuan Lin, Farzad Safaei \\ University of Wollongong, Australia \\ Email: lctran@uow.edu.au
}

\author{
Alfred Mertins \\ University of Lübeck, Germany \\ Email: mertins@isip.uni-luebeck.de
}

\begin{abstract}
Cooperative communications using the Alamouti space-time block code (STBC) has been mentioned intensively. However, to our knowledge, an in-depth error performance analysis for a space-time coded cooperative communications system, where each node acts as both a source node and a relay node for its partner, has not been reported. Intuitively, cooperative communications would not always be better than direct transmission, but only under certain conditions. This paper first derives an exact performance analysis of the Alamouti STBC cooperative communications for a Binary Phase Shift Keying (BPSK) constellation, which could be extended to a higher density modulation scheme. This mathematical analysis facilitates the source nodes to decide pro-actively whether cooperative communications or direct transmission should be used, depending on the channel conditions between the sources themselves and the channel conditions between the sources and the destination. The anterior knowledge of which transmission mode should be used before nodes actually engage in cooperation is useful to keep system operations as simple as possible, while assuring that cooperative communications is definitely beneficial once it is in place. The analytical error performances are then verified by simulations and in-depth discussions on the simulation results are presented.
\end{abstract}

\section{INTRODUCTION}

Relay networks and cooperative communications networks, including the ones using space-time block codes, have been examined intensively in the literature, e.g., [1]- [10].

In [7], the authors successfully derived the exact Bit Error Rate (BER) performance of a relay network (rather than the cooperative network) with either Amplify-and-Forward or soft Decode-and-Forward protocols in an one source-node, tworelay system where the distributed Alamouti Space-Time Block Code (STBC) [11] was implemented. In [8], the authors proposed a relay network with one source and two relays. The source transmits each block of four consecutive symbols to the two relays and to the destination in the first time slot. The relays amplify the received signals and forward them to the destination in the second time slot. Thus this scheme effectively transmits the following orthogonal space-time block code

$$
\left[\begin{array}{cccc}
x_{1} & -x_{2} & -x_{3} & -x_{4} \\
x_{2} & x_{1} & x_{4} & -x_{3} \\
x_{3} & -x_{4} & x_{1} & x_{2}
\end{array}\right] .
$$

The authors then derived the closed-form expression of the system error probability in Rayleigh fading channels. In [9], the authors derived the bit error probability for three transmission mechanisms referred to as Protocols I, II and III in [9], all of which are actually relay networks with one source, one relay and one destination node. In all the above works, the relay nodes might form a space-time block code together with the source node, but they do not act as a source node because they do not transmit their own data. Similarly, in [10], the authors derived the upper (Chernoff) bound of the bit error probability for an one-source, two-relay underwater system that implemented STBCs. Again, this is a relay system rather than a fully cooperative communications system.

To the best of our knowledge, an exact BER performance expression in Rayleigh fading channels has not been derived for a two-source node STBC cooperative network, where each source node acts as both a source and a relay (for its partner). Further, the discussion on the conditions under which cooperative communications is better than a Single-Input SingleOutput (SISO) system (i.e., direct transmission) has not been thoroughly identified. Two questions that could be raised are: a) Is cooperative communications still useful if the Signalto-Noise Ratio (SNR) between the source nodes themselves (referred to as internode channels hereafter) is worse than the SNR between the source nodes and the destination node (referred to as uplink channels)? b) If it is, given an uplink SNR, what is the minimum value of the internode SNR that ensures cooperative communications to be beneficial?

Therefore, in this paper, an exact BER performance analysis of a cooperative communications system in Rayleigh fading is derived for the BPSK modulation which could be extended to an $\mathrm{N}$-ary modulation scheme. The derived BER analysis allows us to answer the above questions theoretically.

The paper is organized as follows. In Section II, our system model and transmission mechanism are introduced. Section III derives the bit error probabilities of a single-input singleoutput (SISO) system and of a $2 \times 1$ Alamouti system in Rayleigh fading channels. Section IV derives the exact bit error probability for our cooperative communications scheme in Rayleigh fading channels. Simulations results are provided in Section V. Section VI concludes the paper. 

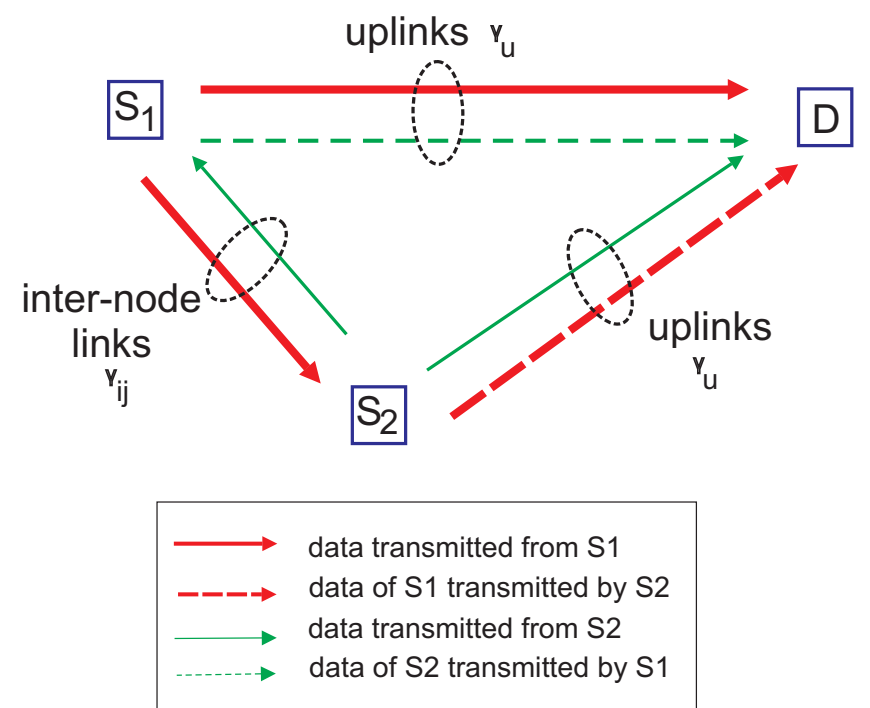

Fig. 1. Three-node Alamouti STBC cooperative communications system.

\section{SYSTEM MODEL}

The system model is depicted in Fig. 1. Channels between the source nodes $S_{1}, S_{2}$ and the destination node $D$ are assumed to be independent, block Rayleigh fading channels. The channel coefficients are modeled as independent complex Gaussian random variables with zero means and unit variances. Additive white Gaussian noise is assumed at all nodes. The transmission is divided into two phases. In the first phase, $S_{1}$ and $S_{2}$ transmit their own symbols $x_{1}$ and $x_{2}$ to $D$ and to each other. In the second phase, $S_{1}$ and $S_{2}$ decode the received signals (we denote the decoded symbols as $x_{1}^{\prime}$ and $x_{2}^{\prime}$ ) and then transmit the conjugate versions $-x_{2}{ }^{*}$ and $x_{1}{ }^{*}$ to $D$, respectively. If the system is half-duplex, i.e., a node cannot transmit and receive signals at the same time, the first phase requires two time slots, thus the whole transmission process requires three time slots. If the system is full-duplex, e.g., in [12], the first phase requires only one time slot, and the whole transmission process requires two time slots. The analysis in this paper holds, regardless of whether the system is halfduplex or full-duplex. During the two phases, the following Alamouti code [11]

$$
\left[\begin{array}{cc}
x_{1} & x_{2} \\
-x_{2}^{\prime *} & x_{1}^{\prime *}
\end{array}\right]
$$

will be transmitted from $S_{1}$ and $S_{2}$ to $D$. The channel coefficients are assumed to remain constant during the two phases, but change randomly after each Alamouti code block. Further, we denote $\bar{\gamma}_{i j}$ and $\bar{\gamma}_{u}$ as the average SNRs of the internode links (between $S_{i}$ and $S_{j}$ ) and the uplinks, respectively. For simplicity, we consider the BPSK modulation throughout this paper.

\section{BER of SISO AND Alamouti STBC}

To formulate the BER expression of our cooperative communications system, we first derive the following two results.

\section{Result 1: BER expression of a SISO system}

In the first phase, $S_{1}$ and $S_{2}$ need to exchange their messages in order to establish the Alamouti STBC. These transmissions can be treated as a Single-Input Single-Output (SISO) system. Thus the BER of the transmission between $S_{i}$ and $S_{j}$, denoted as $\mathrm{BER}_{i j}$, for a BPSK modulation in Rayleigh fading channels is [13, Eq. (20)]

$$
B E R_{i j}=P_{i j}=\frac{1}{2}\left(1-\sqrt{\frac{\bar{\gamma}_{i j}}{1+\bar{\gamma}_{i j}}}\right) .
$$

\section{Result 2: BER expression of the Alamouti STBC system}

This result derives the BER of an Alamouti STBC communications system. As mentioned in [11], the diversity order of a two-transmit antenna, one-receive antenna Alamouti STBC system is equal to that of the Maximum Ratio Combing (MRC) technique with one transmit antenna and two receive antennas. However, to make a fair comparison between the two schemes, the transmission power from each of the two transmit antennas in the STBC case must be scaled down by a factor of 2 to achieve the same total transmission power as that transmitted from the single antenna in the MRC case. Consequently, the SNR per bit in the STBC case is half that value in the MRC case.

The error probability of the MRC system with one transmit antenna and two receive antennas is calculated as [14, Eq.(14.4-15)]

$$
P_{M R C}=\frac{1}{4}(1-\mu)^{2}(2+\mu),
$$

where

$$
\mu=\sqrt{\frac{\gamma}{1+\gamma}},
$$

and $\gamma$ is the average SNR per bit of the links between the transmit antenna and the two receive antennas.

Therefore, the error probability for the two-transmit, onereceive antenna Alamouti STBC system in Rayleigh fading channels, denoted as $\mathrm{P}_{A}$, can be expressed as

$$
P_{A}=\frac{1}{4}(1-p)^{2}(2+p),
$$

where

$$
p=\sqrt{\frac{\bar{\gamma}_{u} / 2}{1+\bar{\gamma}_{u} / 2}}=\sqrt{\frac{\bar{\gamma}_{u}}{2+\bar{\gamma}_{u}}},
$$

and $\bar{\gamma}_{u}$ is the average SNR of the uplink channels and the scale factor of 2 is used for a fair comparison with the SISO system to guarantee the same total transmission powers in both cooperative and SISO systems. We can rewrite $P_{A}$ as

$$
P_{A}=\frac{1}{4}\left[1-\sqrt{\frac{\bar{\gamma}_{u}}{2+\bar{\gamma}_{u}}}\right]^{2}\left[2+\sqrt{\frac{\bar{\gamma}_{u}}{2+\bar{\gamma}_{u}}}\right] .
$$

Equations (1) and (5) will be used in our performance analysis in the next section. 


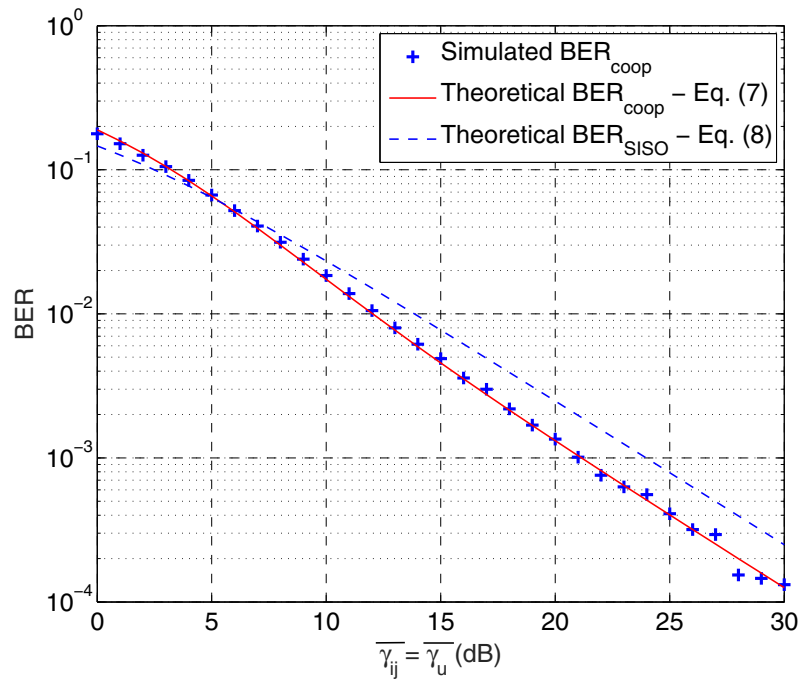

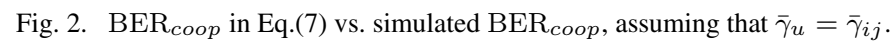

\section{EXACT BER EXPRESSION OF ALAMOUTI STBC COOPERATIVE COMMUNICATIONS}

Let us denote the BER of the cooperative communications as $\mathrm{BER}_{\text {coop }}$. Since decoding at the source nodes might be imperfect, to derive the exact $\mathrm{BER}_{\text {coop }}$, we split the case of erroneous reception at the destination into the following two complementary cases.

Case 1: Errors occur in the uplinks (regardless of whether the internode links are correct or not). Intuitively, the bit error probability for this case, denoted as $\mathrm{BER}_{1}$, is at most equal to that of the two-transmit antenna Alamouti STBC, i.e., $\mathrm{BER}_{1}=P_{A}$.

Case 2: No errors occur in the uplink transmission. The probability for this case is $P_{2}=1-P_{A}$. The following four random events may occur in this case: transmissions from $S_{1}$ to $S_{2}$ (hereafter denoted as $S_{1} \rightarrow S_{2}$ ) and transmission from $S_{2}$ to $S_{1}\left(S_{2} \rightarrow S_{1}\right)$ are both incorrect; $S_{1} \rightarrow S_{2}$ and $S_{2} \rightarrow S_{1}$ are both correct; $S_{1} \rightarrow S_{2}$ is correct and $S_{2} \rightarrow S_{1}$ is incorrect; and $S_{1} \rightarrow S_{2}$ is incorrect and $S_{2} \rightarrow S_{1}$ is correct. It is reasonable to assume that these four random events are equiprobable, i.e., $P_{E_{i}}=0.25$, for $i=1, \ldots, 4$. The BER in each event can be calculated as follows.

- Event $E_{1}: S_{1} \rightarrow S_{2}$ and $S_{2} \rightarrow S_{1}$ are both incorrect. The bit error probability for two BPSK symbols being simultaneously erroneous is $P_{i j}^{2}$. Two erroneous BPSK symbols cause two bit errors in the destination node (note that the uplinks are correct). Thus, the bit error rate caused by this event is $\mathrm{BER}_{E_{1}}=2 \mathrm{BER}_{i j}=$ $2 P_{i j}^{2}$.

- Event $E_{2}: S_{1} \rightarrow S_{2}$ and $S_{2} \rightarrow S_{1}$ are both correct. This case does not cause any error at the destination, thus $\mathrm{BER}_{E_{2}}=0$.

- Event $E_{3}: S_{1} \rightarrow S_{2}$ is correct and $S_{2} \rightarrow S_{1}$ is incorrect. The bit error rate caused by event $E_{3}$ is represented as $\mathrm{BER}_{E_{3}}=P_{i j}$.

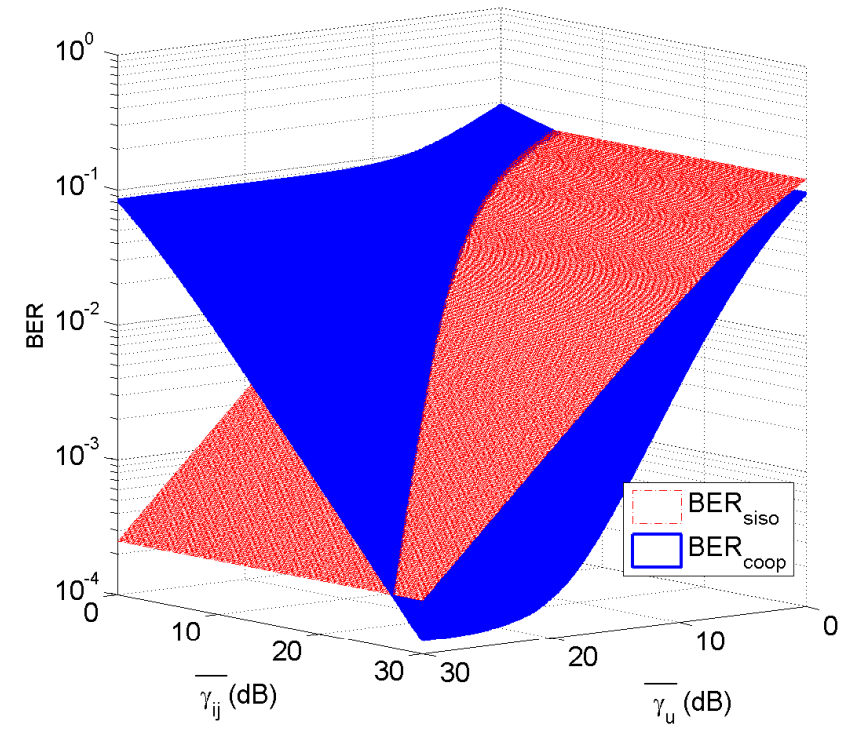

Fig. 3. BER Boop $_{\text {in Eq.(7) vs. BER }}$ siso in Eq.(8) for arbitrary $\bar{\gamma}_{i j}$ and $\bar{\gamma}_{u}$ in the $[0,30] \mathrm{dB}$ range.

- Event $E_{4}: S_{1} \rightarrow S_{2}$ is incorrect and $S_{2} \rightarrow S_{1}$ is correct. Similarly to the event $E_{3}$, we have $\mathrm{BER}_{E_{4}}=$ $P_{i j}$.

Hence, the BER of Case 2 is

$$
\begin{aligned}
B E R_{2} & =\left(1-P_{A}\right) \sum_{i=1}^{4} P_{E_{i}} \mathrm{BER}_{E_{i}} \\
& =0.5\left(1-P_{A}\right)\left(P_{i j}^{2}+P_{i j}\right) .
\end{aligned}
$$

As a result, the BER of the cooperative system is

$$
\mathrm{BER}_{\text {coop }}=\sum_{k=1}^{2} \mathrm{BER}_{k}=P_{A}+0.5\left(1-P_{A}\right)\left(P_{i j}^{2}+P_{i j}\right) .
$$

Replacing (1) and (5) into (6), we have

$$
\begin{aligned}
& \operatorname{BER}_{\text {coop }}=\frac{1}{4}\left[1-\sqrt{\frac{\bar{\gamma}_{u}}{2+\bar{\gamma}_{u}}}\right]^{2}\left[2+\sqrt{\left.\frac{\bar{\gamma}_{u}}{2+\bar{\gamma}_{u}}\right]}\right. \\
& \times \quad\left[1-\frac{1}{4}\left(1-\sqrt{\frac{\bar{\gamma}_{i j}}{1+\bar{\gamma}_{i j}}}\right)-\frac{1}{8}\left(1-\sqrt{\frac{\bar{\gamma}_{i j}}{1+\bar{\gamma}_{i j}}}\right)^{2}\right] \\
& +\frac{1}{8}\left(1-\sqrt{\frac{\bar{\gamma}_{i j}}{1+\bar{\gamma}_{i j}}}\right)\left(3-\sqrt{\frac{\bar{\gamma}_{i j}}{1+\bar{\gamma}_{i j}}}\right) .
\end{aligned}
$$

Clearly, the bit error probability of the cooperative communications dependents on both the internode SNR, $\bar{\gamma}_{i j}$, and the uplink SNR, $\bar{\gamma}_{u}$. For a given $\bar{\gamma}_{u}$, the value of $\bar{\gamma}_{i j}$ could significantly affect the system performance (and vice versa). If $\bar{\gamma}_{i j}$ is lower than a threshold value, denoted as $\bar{\gamma}_{t h}$, cooperative communications might be inferior, compared to a SISO system.

To find the threshold value, we equate $\mathrm{BER}_{\text {coop }}$ to that of

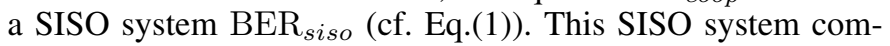
prises one source node (either $S_{1}$ or $S_{2}$ ) and the destination 


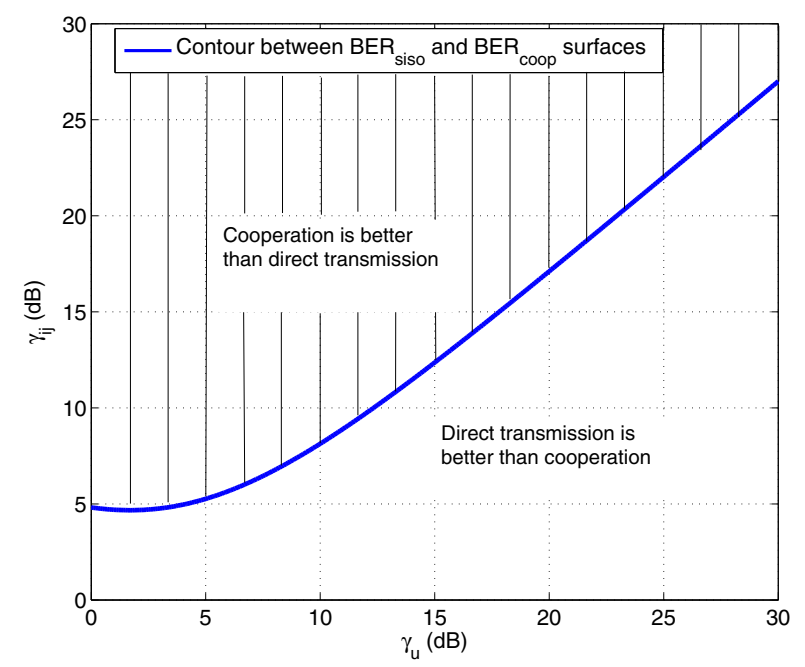

Fig. 4. The contour between $\mathrm{BER}_{\text {coop }}$ and $\mathrm{BER}_{\text {siso }}$ surfaces indicates possible values of the threshold SNR $\bar{\gamma}_{t h}$ and shows the usefulness of cooperative communications.

node, and it has the average SNR value $\bar{\gamma}_{u}$.

$$
\mathrm{BER}_{\text {siso }}=\frac{1}{2}\left(1-\sqrt{\frac{\bar{\gamma}_{u}}{1+\bar{\gamma}_{u}}}\right) .
$$

For a given $\bar{\gamma}_{u}$, from Eqs. (7) and (8), we can calculate the threshold value $\bar{\gamma}_{t h}$. If $\bar{\gamma}_{i j}$ is lower than $\bar{\gamma}_{t h}$, the system may give up cooperation and use direct transmission instead to gain better performance. More details on this discussion are given in the next section.

\section{Simulations AND Discussions}

In this section, we verify the validity of our performance analysis mentioned in (7) and provide in-depth discussions on the performance and usefulness of the cooperative communications system in comparison with the direct transmission system. In particular, Fig. 2 shows the agreement of BER $\mathrm{Boop}_{\text {co }}$ achieved by a Monte-Carlo simulation and BER $_{\text {coop }}$ calculated by (7), assuming that $\bar{\gamma}_{i j}=\bar{\gamma}_{u}=0, \ldots, 30 \mathrm{~dB}$. This agreement confirms the validity of Eq. (7).

Fig. 2 also plots the theoretical bit error performance of an SISO system (cf. Eq. (8)), assuming that the SNR in this SISO system is $\bar{\gamma}_{u}$ (thus $\bar{\gamma}_{i j}$ ). In this scenario, the cooperative communication system provides better performance by about $3 \mathrm{~dB}$ at BER $=10^{-3}$ and $3.5 \mathrm{~dB}$ at BER $=10^{-4}$, compared to the SISO system, without any increase of the total transmission power. It is clear that the diversity order of the cooperative communication system is higher than that in the SISO system, and that the full diversity order of two cannot be achieved in a realistic cooperative communication system due to the possible erroneous information exchange between the source nodes. It is important to emphasize that these observations have been derived for the case $\bar{\gamma}_{i j}=\bar{\gamma}_{u}$. The cooperative communication might be much better than the SISO system in some other cases as shown later in Fig. 3 .

Fig. 3 compares $\mathrm{BER}_{\text {coop }}$ in (7) and $\mathrm{BER}_{\text {siso }}$ in (8) for arbitrary values of $\bar{\gamma}_{i j}$ and $\bar{\gamma}_{u}$ in the range $[0,30] \mathrm{dB}$. From this

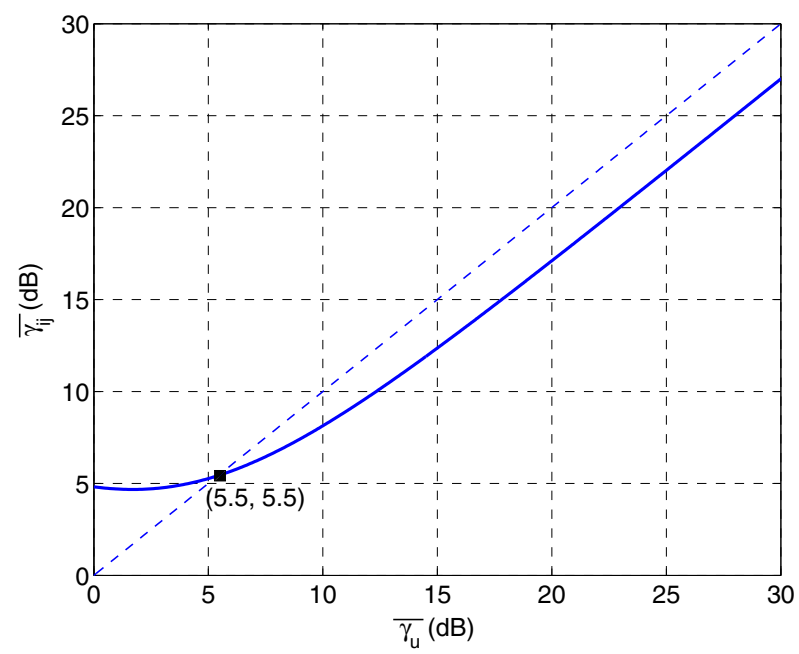

Fig. 5. The contour between $\mathrm{BER}_{\text {coop }}$ and $\mathrm{BER}_{\text {siso }}$ surfaces indicates the relation between the magnitudes of $\bar{\gamma}_{i j}$ and $\bar{\gamma}_{u}$ to make cooperative communications useful.

figure, one can see clearly that cooperative communications is only better than than SISO (i.e. direct transmission between the source node and the destination) in the region where the $\mathrm{BER}_{\text {coop }}$ surface goes under the $\mathrm{BER}_{\text {siso }}$ surface. Clearly, the cooperative communication system could be much better than the direct transmission system (i.e., the $\mathrm{BER}_{\text {coop }}$ surface is deep under the $\mathrm{BER}_{\text {siso }}$ surface), especially, when the internode channels have a large $\bar{\gamma}_{i j}$ value (e.g., when $\bar{\gamma}_{i j}=30 d B$ and $\bar{\gamma}_{u}=15, \ldots, 30 \mathrm{~dB}$ ).

Fig. 4 presents the contour of the intersection between $\mathrm{BER}_{\text {coop }}$ and $\mathrm{BER}_{\text {siso }}$ surfaces. This contour presents the set of possible values of the threshold $\bar{\gamma}_{t h}$. We recall that, for a certain value of $\bar{\gamma}_{u}$, the cooperative communications is only better than the direct transmission if $\bar{\gamma}_{i j}>\bar{\gamma}_{t h}$. For instance, given the uplink SNR $\bar{\gamma}_{u}=15 \mathrm{~dB}$ corresponding to the threshold SNR $\bar{\gamma}_{t h}=12.3 \mathrm{~dB}$, cooperative communications will be beneficial if the internode SNR $\bar{\gamma}_{i j}$ is greater than 12.3 $\mathrm{dB}$. On the contrary, given the internode SNR $\bar{\gamma}_{i j}=15 \mathrm{~dB}$ for instance, cooperative communications will be better than noncooperative communications if the uplink SNR $\bar{\gamma}_{u}$ is less than $17.7 \mathrm{~dB}$ (if $\bar{\gamma}_{u}$ is greater than this value, direct transmission would be preferable). More generally, cooperative communications starts to bring benefit over the direct transmission if the point presented by the coordinates $\left(\bar{\gamma}_{u}, \bar{\gamma}_{i j}\right)$ is in the marked area (above the contour).

In Fig. 5, the dotted line presenting the equality $\bar{\gamma}_{i j}=\bar{\gamma}_{u}$ is included. The intersection between this line and the aforementioned contour is found to be the point $(5.5 \mathrm{~dB}, 5.5 \mathrm{~dB})$, which are the root of the equation $\mathrm{BER}_{\text {coop }}=\mathrm{BER}_{\text {siso }}$ with the condition $\bar{\gamma}_{i j}=\bar{\gamma}_{u}$. (The intersection at $5.5 \mathrm{~dB}$ can also be seen in Fig. 2). From Fig. 5, one can observe that the threshold SNR $\bar{\gamma}_{t h}>\bar{\gamma}_{u}$ if $\bar{\gamma}_{u}<5.5 \mathrm{~dB}$ (the contour is above the dotted line), and $\bar{\gamma}_{t h}<\bar{\gamma}_{u}$ otherwise (the contour is below the dotted line). This means that when $\bar{\gamma}_{u}$ is small (less than $5.5 \mathrm{~dB}$ ), the internode SNR must be higher than the uplink SNR in order for cooperative communications to be better than SISO. However, for $\bar{\gamma}_{u}>5.5 \mathrm{~dB}, \gamma_{t h}$ is always smaller than $\bar{\gamma}_{u}$, i.e., cooperative communications might still 
be better than SISO, even when the internode SNR is smaller than the uplink SNR. This is because the decoding processes at all source and destination nodes become more accurate when the internode and uplink channels have better quality, thus the diversity of the Alamouti STBC cooperative communications between nodes starts to bring about significant benefits.

Another observation from Figs. 4 and 5 is that, given a certain value of the uplink SNR, direct transmission is better than cooperative communications at a lower range of the internode SNR, while the reverse is true if the internode SNR is higher than a certain value. Interestingly, a similar observation was reported in [8, Figs.3-5] for the orthogonal STBC mentioned in Section I, although our system model, space-time codes, cooperation mechanism and performance analysis approach are totally different from those in [8].

In summary, if the uplink SNR $\bar{\gamma}_{u}$ is known at the source nodes, from Fig. 4 or 5 , we can calculate the threshold value $\bar{\gamma}_{t h}$ and vice versa. If the source nodes also know the internode SNR $\bar{\gamma}_{i j}$, they could decide if cooperative communications should be used (if $\bar{\gamma}_{i j}>\bar{\gamma}_{t h}$ ), or direct transmission should be used instead.

\section{Conclusions}

This paper derives the exact performance analysis of a binary space-time block coded cooperative communications system using the Alamouti code in Rayleigh fading channels. In-depth discussions on the performance as well as the usefulness of cooperative communications compared to the direct transmission are also presented. The exact error performance analysis allows source nodes to have an anterior knowledge of which transmission mode should be used, thus allowing nodes to engage in cooperation only when it is beneficial. This is useful in a number of applications to keep the system design and operations as simple as possible, while assuring that cooperative communications is definitely beneficial once it is in place.

Our future work is to extend the performance analysis in this paper to an $\mathrm{N}$-ary space-time block coded cooperative communications system with multiple receive antennas at each node in Rician fading channels.

\section{ACKNOWLEDGEMENTS}

The first author sincerely thanks the Alexander von Humboldt (AvH) Foundation, Germany, for its support of this work under the Postdoctoral Further Research Stay Fellowship.

\section{REFERENCES}

[1] A. Sendonaris, E. Erkip, and B. Aazhang, "User cooperation diversity part I: system description", IEEE Trans. Commun., vol. 51, no. 11, pp. 1927-1938, Nov. 2003.

[2] T. E. Hunter, and A. Nosratinia, "Diversity through coded cooperation", IEEE Trans. Wireless Commun., vol. 5, no. 2, pp. 283-289, Feb. 2006,

[3] H.-Q. Lai, and K.J.R. Liu, "Space-time network coding", IEEE Trans. Sign. Process., vol. 59, no. 4, pp. 1706-1718, April 2011.

[4] Z. Lin, L. C. Tran, and F. Safaei, "Performance evaluation of cooperative communications for STFC MB-OFDM UWB", in Proc. 13rd IEEE Int. Symp. Comp. Inform. Technol. (ISCIT 2013), Sept. 2013, pp. 46-51.

[5] Z. Lin, L. C. Tran, and F. Safaei, "Order-4 quasi-orthogonal cooperative communication in STFC MB-OFDM UWB", in Proc. 6th IEEE Int. Conf. Sign. Process. Commun. Syst. (ICSPCS 2012), Dec. 2012, Gold Coast, Australia.

[6] Z. Lin, L. C. Tran, and F. Safaei, "Order-4 orthogonal cooperative communication in space-time-frequency coded MB-OFDM UWB", in Proc. 12nd IEEE Int. Symp. Comp. Inform. Technol. (ISCIT 2012), Oct. 2012, pp. 920-925.

[7] P. Huo, and L. Cao, "Distributed STBC with soft information relay based on Gaussian approximation", IEEE Sign. Process. Lett., vol. 19, no. 10, pp. 599-602, 2012.

[8] H. Y. Kong, and H. V. Khuong, "Performance analysis of spacetime block coded cooperative wireless transmission in Rayleigh fading channels", Journal of Communications and Networks, vol. 8, no. 3, pp. 306-312, Sept. 2006

[9] J. He, and P. Y. Kam, "Exact bit error probability of cooperative spacetime block coding with amplify-and-forward strategy", in Proc. IEEE International Conference on Communications (ICC '08), May 2008, pp. 4591-4595.

[10] M. Vajapeyam, S. Vedantam, U. Mitra, J. C. Preisig, and M. Stojanovic, "Distributed spacetime cooperative schemes for underwater acoustic communications", IEEE J. Oceanic Eng., vol. 33, no. 4, pp. 489-501, Oct. 2008

[11] S. M. Alamouti, "A simple transmit diversity technique for wireless communications", IEEE J. Select. Areas Commun., vol. 16, no. 8, pp. 1451-1458, Oct. 1998

[12] L. C. Tran, A. Mertins, and T. A. Wysocki, "Cooperative communication in space-time-frequency coded MB-OFDM UWB", in Proc. 68th IEEE VTC2008-Fall, 2008, pp. 1-5.

[13] W. C. Lindsey, "Error probabilities for Rician fading multichannel reception of binary and $\mathrm{N}$-ary signals", IEEE Trans. Inform. Theory, vol. IT-10, pp. 339-350, Oct. 1964.

[14] J. G. Proakis, Digital communications, 4th Ed., McGraw-Hill, Boston, 2001 